\title{
Modelling of impurity deposition in gaps of castellated surfaces with the 3D-GAPS code
}

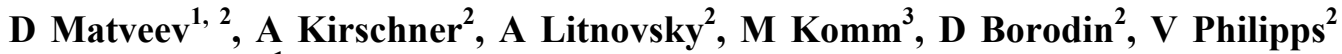 \\ and $G$ Van Oost ${ }^{1}$ \\ ${ }^{1}$ Department of Applied Physics, Ghent University, Plateaustraat 22, B-9000 Ghent, \\ Belgium \\ ${ }^{2}$ Institut für Energieforschung - Plasmaphysik, Forschungszentrum Jülich GmbH, \\ Association EURATOM-FZJ, Trilateral Euregio Cluster, D-52425 Jülich, Germany. \\ ${ }^{3}$ Department of Surface and Plasma Science, Charles University, CZ-18000 Prague, \\ Czech Republic
}

E-mail: d.matveev@fz-juelich.de

\begin{abstract}
The Monte-Carlo neutral transport code 3D-GAPS is described. The code models impurity transport and deposition in remote areas, such as gaps between cells of castellated plasma-facing surfaces. A step-by-step investigation of the interplay of different processes that may influence the deposition inside gaps, namely particle reflection, elastic neutral collisions, different particle sources, chemical erosion, and plasma penetration into gaps is presented. Examples of modelling results in application to the TEXTOR experiment with a castellated test limiter are provided. It is shown that only with the assumption of the presence of species with different reflection probabilities, simulated carbon deposition profiles agree with experimental observations for side surfaces of the gaps. These species can be attributed to different particle sources, e.g., carbon atoms and hydrocarbon radicals. Background carbon ions and atoms have low and moderate values of the reflection coefficient $(\mathrm{R} \leq 0.6)$, while some of hydrocarbon radicals produced by chemical erosion of redeposited carbon layers have high reflection probability $(\mathrm{R} \geq 0.9)$. Deposition at the bottom of the gaps can not be adequately reproduced unless extreme assumptions on particle sources and reflection properties are imposed. Elastic neutral collisions and ionization of neutrals escaping the gaps have no significant influence on the results. Nevertheless, Particlein-Cell simulations of plasma penetration into gaps are essential for estimating the incoming ion flux and lead to a better quantitative agreement with experimental observations.
\end{abstract}

PACS: 52.40.Hf; 52.25.Vy; 52.65.Pp

\section{Introduction}

Plasma-material interactions are of great importance for next step fusion devices such as ITER [1], [2]. Intensive particle and heat loads lead to material erosion, thus decreasing the lifetime of plasma facing components [3]. Eroded material is transported elsewhere in the device where it is likely to be deposited. As a result, mixed materials with poorly known thermal and mechanical properties [4] and amorphous redeposited layers with a strong potential for radioactive fuel (tritium) retention and dust formation [3] may be formed. Carbon-based materials selected for ITER divertor targets in the nonactivated phase due to their outstanding thermo-mechanical properties are prone to chemical erosion by hydrogen atoms and corresponding migration mechanisms [5]. Experiments show that hydrogenrich carbon layers are mainly formed on plasma shadowed areas and especially in narrow gaps between surface cells of castellated structures [6], which can not be effectively accessed by most of the proposed tritium-removal techniques [7]. Thus, understanding of impurity transport and deposition inside gaps is of crucial importance. 
Several experimental and modelling attempts have been made to study material deposition and hydrogen retention in gaps in present-day fusion devices [8]-[13]. All experiments show maximal carbon deposition at the plasma-nearest edge inside gaps with a strong decrease of the deposited amount more deeply in the gaps, which in most cases can be approximated as a double exponential decay. Experiments with an ITER-like castellated test limiter performed at TEXTOR [9] showed that not only side surfaces but also the bottom of gaps can accumulate significant carbon deposits. Gap shaping eliminating leading edges was proposed as a possible solution to mitigate deposition in gaps by restricting the fluxes of particles capable of entering the gap. Meanwhile in DIII-D experiments it was demonstrated that the hydrogen fraction in deposited layers in gaps can be also significantly reduced if the material temperature is increased [10]. Such an observation indicates that hydrogen recycling (desorption) and chemical processes (erosion) are important for assessing deposition in gaps. Modelling can only partly reproduce the experimental observations and therefore the present picture of impurity transport in gaps is not yet clear enough to make predictions for ITER.

In this paper, the problem of carbon deposition in gaps is addressed by means of modelling with the Monte-Carlo code 3D-GAPS, which is described in section 2. Different processes that may affect the deposition are examined in successive steps in section 3 with results of the modelling validated against the experimental data from TEXTOR. Section 4 gives summary and outlook.

\section{The 3D-GAPS code}

The Monte-Carlo neutral transport code 3D-GAPS uses the test particle approach to describe transport of impurity atoms in three-dimensional geometry (3D). The code is flexible to support arbitrarily defined volumes with plain boundaries and therefore is suitable for modelling of shadowed and remote areas. In the present investigations, the simulation volume is optimized for a gap-like geometry. Neutral particles are launched from certain source locations inside the defined simulation volume referenced as a gap. Under the assumption of no or negligible plasma inside the gap, neutrals move along straight lines until they reach one of limiting surfaces within the gap or experience a collision. The limiting surface can be represented either by a real gap wall or by an open surface. In the latter case, particles are considered to be lost from the gap after having crossed such an open surface. For a rectangular gap, the top of the gap (the gap entrance plane) defines the loss surface. If a particle reaches a real surface, it may be either reflected or deposited. The reflection probability is given by pre-defined reflection coefficients dependent on the projectile and material combination. An analytical function can be introduced to describe the dependence of the reflection coefficient on the incident angle and energy. For reflected particles, the cosine angular distribution is assumed; however for parameter studies specular reflection has also been utilized. Interaction of test particles with residual gas molecules (elastic neutral collisions) can be taken into account. In this case, the particle trajectory is deflected after each collision. Plasma penetration into the gap can be taken into account by coupling with Particle-in-Cell (PIC) simulations as described in section 2.3. The entire simulation is split into simulation time steps, $\Delta t$ (typically $\sim 0.1 \mathrm{~s}$ ), during which a finite number of test particles is simulated until they are lost or deposited at any gap surface. The deposited particles are taken into account to update the local surface content after each simulation time step. As output information, 2D distributions of deposited amounts and local surface concentrations for different species are stored for each surface within the gap. In the following subsections, certain code features are described in more detail.

\subsection{Particle sources and corresponding fluxes}

Two different types of particle sources are considered: background plasma sources and surface erosion sources. Background plasma sources provide particles launched from a certain surface (including open surfaces). For instance, these can be plasma ions (hydrogen or impurities) reflected as neutrals from the plasma-wetted area. Erosion sources account for physical sputtering and chemical erosion processes. Each test particle embodies a certain amount of real particles. In case of background plasma source, this amount is pre-defined by the ratio of the incoming particle flux to the number of test particles. For an erosion source, it is calculated according to the amount of eroded particles, $N_{\text {eroded }}$, 
and a given number of test particles. In the so-called homogeneous mixing model (HMM) described in the next section and used to evaluate local concentrations of elements in the surface it is assumed:

$$
N_{\text {eroded }}=Y \times \Gamma \times A \times \Delta t \times c_{i}
$$

Here $Y$ gives the pre-defined erosion/sputtering yield; $\Gamma$ represents the flux of particles causing erosion (e.g., hydrogen isotopes in case of chemical erosion of carbon) onto the local surface of area $A$; and $c_{i}$ stands for the local surface concentration of the eroded element (e.g., carbon).

An external source of particles offering possibilities of iterative data exchange with other impurity transport codes, such as ERO [14], can be set in addition to the source types described above. In this case, a list of test particles initializing their positions, velocities, types, etc has to be directly provided to the code. Similarly, a list of particles escaping the gap can be then provided as an output from the 3D-GAPS code.

\subsection{Homogeneous surface mixing model (HMM)}

In the frame of the HMM approximation, only the top layer of the surface takes part in the interaction processes. Different species are distributed homogeneously inside this interaction layer, and the total number of particles in the layer is kept constant. The excess number of particles that may appear due to net deposition is moved to the bulk in accordance to relative concentrations of different species in the interaction layer. The number of particles that may be lacking due to net erosion is filled up with particles from the bulk, which serves as a reservoir of an infinite number of substrate particles.

Generally, the interaction layer thickness (ILT) represents an effective depth for which the surface content is strongly affected by erosion/deposition processes. It is determined to a large extent by the effective penetration depth of incident particles, but also the balance between erosion and deposition rates is important. In the frame of the HMM approximation, ILT is a free parameter directly connected with the simulation time step. Thus, the steady state condition (concentrations of different species in the layer do not change with time anymore) is reached faster with smaller ILT. It should be noted that the resulting equilibrium concentrations of species in the interaction layer are independent of the choice of ILT and are determined exclusively by the balance of incoming and outgoing particle fluxes. Nevertheless, one should take care that both the number of eroded particles and the number of deposited particles in a single simulation time step remain well below the total number of particles in the interaction layer. Total eroded/deposited amounts can be calculated from the steady-state erosion/deposition rates, provided the steady state condition is reached much faster compared to the total plasma exposure time, what is usually the case.

\subsection{Coupling with Particle-in-Cell simulations}

The 2D in space and 3D in velocity SPICE2 (Sheath Particle in Cell) code [15] uses the PIC technique to simulate self-consistently plasma penetration into a gap. Variable geometry with arbitrary number of rectangular, triangular and circular objects can be implemented. Injection of particles is performed respecting the orientation of the magnetic field, which is assumed to be uniform. Output information from SPICE2 contains 2D distributions of plasma parameters, such as the electron and ion temperatures and densities, flow velocities, and electric field components. Additionally, the distribution of the ion flux over the surfaces inside the gap can be calculated. This information can be used in the 3D-GAPS code to adjust particle fluxes for background plasma sources and to account for ionization of neutrals leaving the gap. A robust automatic fitting procedure has been developed for flux functions delivered by the SPICE2 code to facilitate their use in the 3D-GAPS code.

\subsection{Elastic neutral collisions}

The hydrogen influx into the gap of a closed geometry with subsequent recycling and release of $\mathrm{H}_{2}$ molecules should lead to a build-up of neutral pressure inside the gap. Test particles crossing the gap may undergo scattering collisions with such residual molecules. Elastic neutral collision can be treated in the 3D-GAPS code using pre-calculated collision rates as in the ERO code [16]. Elastic collision rates are calculated based on momentum transfer cross-section data and approximate asymptotic energy dependencies. In case of a collision, the velocity of a hydrogen molecule is randomly generated 
with Maxwell distribution for a given gas temperature. The oppositely directed velocities of the test particle and the molecule after the collision are assumed to be isotropically random in the centre of mass system.

\subsection{Treatment of elementary processes}

The simulation for a single test particle is divided into integration time steps, $\mathrm{d} t$ (typically $<10^{-7} \mathrm{~s}$ ). Neutral atoms feel no forces and move along straight lines. If a particle becomes ionized, it feels the Lorentz force and its motion is calculated using the Boris method [17] that separates the electric and magnetic forces completely. For random processes, such as elastic neutral collisions and electron impact ionization, the probability of collision or ionization is evaluated at each time step according to reaction rates dependent on local gas and plasma parameters. Reaction rates for elastic collision and ionization processes are provided by the ERO code database which, in particular, uses ADAS data [18]. Whether an event of collision or ionization happens or not is decided by the Monte-Carlo approach, i.e., comparing the probability of the event to happen with a random number uniformly distributed between 0 and 1 .

\section{Parameter studies and comparison with experimental results from TEXTOR}

\subsection{Experiment in TEXTOR}

A direct comparative investigation of the effect of gap shaping on deposition inside gaps was performed in TEXTOR [8]. A double-roof castellated tungsten test limiter with two cell geometries, non-shaped (rectangular) and roof-like shaped (figure 1), was exposed in the scrape-off-layer (SOL) plasma for 16 repetitive NBI-heated discharges with total plasma duration of about 112 seconds. The edge plasma parameters during the experiment were measured with the fast probe and He-beam diagnostics. The bulk temperature of the limiter was monitored with thermocouples and was in the range of $200-250^{\circ} \mathrm{C}$. In the end of exposure, problems with plasma control caused plasma shifts towards the limiter and led to temperature excursions at the plasma-closest edge of the castellation up to $1500^{\circ} \mathrm{C}$. Post-mortem surface and elemental analyses were made inside the gaps, on plasma-facing top surfaces and on the gap holder as described in [9]. Deposits with up to $200 \mathrm{~nm}$ thickness were observed at the bottom of gaps compared to up to $500 \mathrm{~nm}$ thick deposits on the side surfaces. Up to $14 \%$ of the total amount of carbon deposited in poloidal gaps was found at their bottom. Poloidal rectangular and shaped gaps of this experiment have been modelled with the 3D-GAPS code.
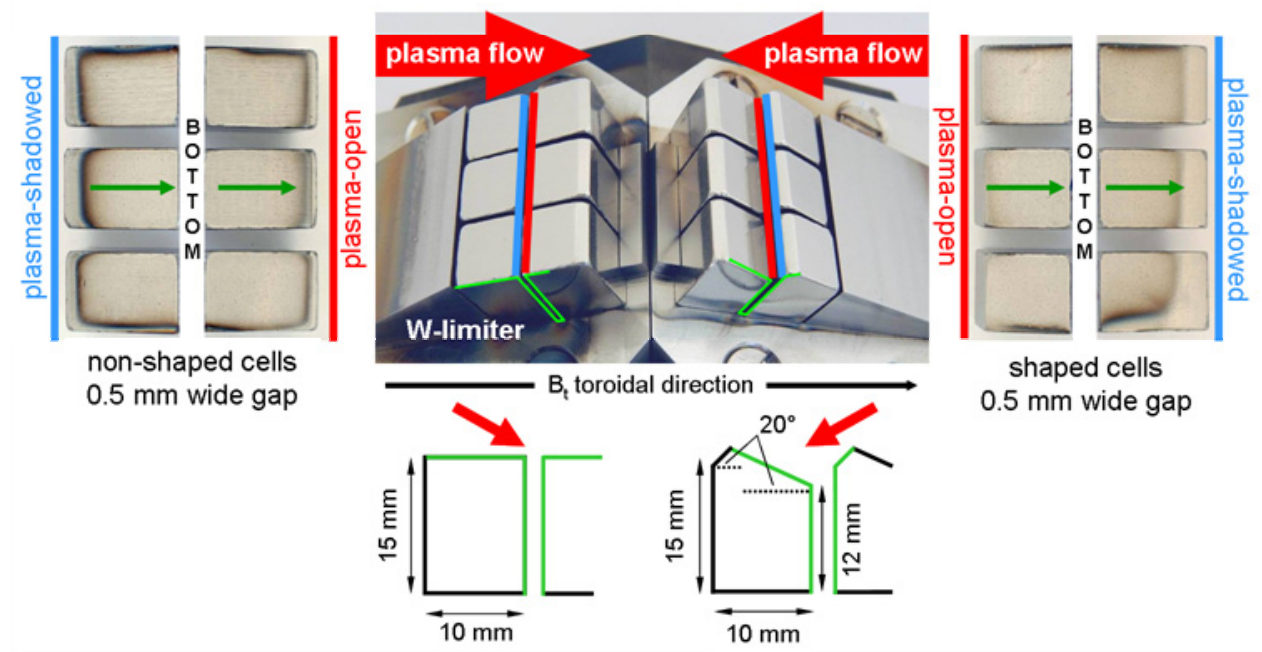

Figure 1. Photographic view of the test limiter (middle) and side views of castellation cells after disassembling representing unfolded views of poloidal rectangular (left) and shaped (right) gaps. 


\subsection{Setup of the modelling}

Figure 2 illustrates geometries of poloidal gaps used in the modelling. Different sources of particles capable of entering the gap are schematically depicted in the same figure.
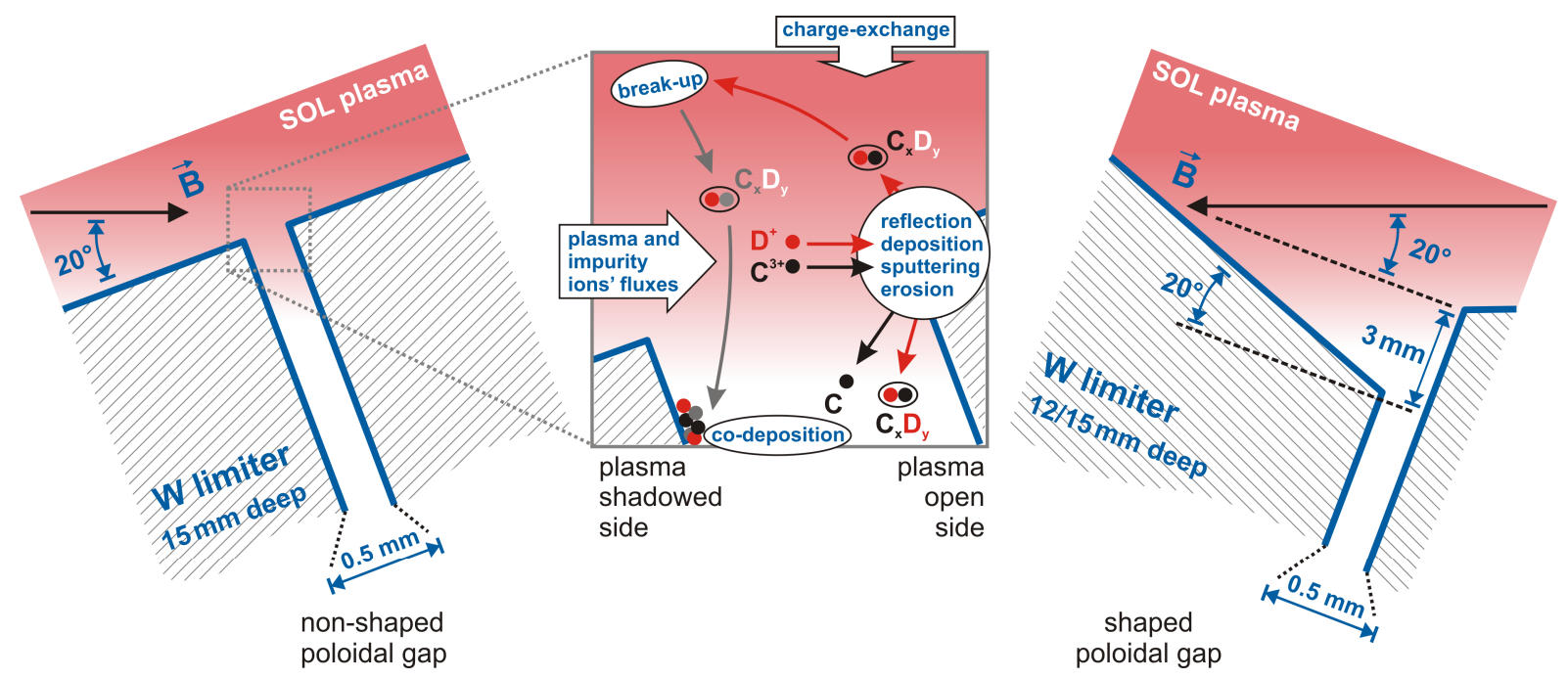

Figure 2. Geometries of poloidal gaps used in the modelling. The gaps are $15 \mathrm{~mm}$ (non-shaped) and $15 / 12 \mathrm{~mm}$ (shaped) deep with a width of $0.5 \mathrm{~mm}$.

The ion flux distribution over gap surfaces was obtained from SPICE2 simulations for the plasma parameters near the castellation corresponding to fast probe measurements $\left(T_{e}=20 \mathrm{eV}\right.$, $n_{e}=6 \times 10^{12} \mathrm{~cm}^{-3} ; T_{i}=1.5 T_{e}$ [19]). Due to a complex potential structure formed near the gap entrance (figures $3 \mathrm{a}$ and $3 \mathrm{~b}$ ), incoming plasma ions have certain probabilities to be repelled from the gap or to hit the so-called plasma-shadowed side of the gap. For the rectangular gap, roughly $20 \%$ of incoming ions are repelled from the gap; approximately $5 \%$ of particles reaching the gap surfaces land on the plasma-shadowed side. Resulting distributions for rectangular and shaped gaps are shown in figures $4 \mathrm{c}$ and $4 \mathrm{~d}$. Sides denoted by letters $\mathrm{C}$ and $\mathrm{D}$ are called plasma open; sides denoted by letter A are plasma shadowed. Side $\mathrm{P}$ is parallel to the magnetic field lines.
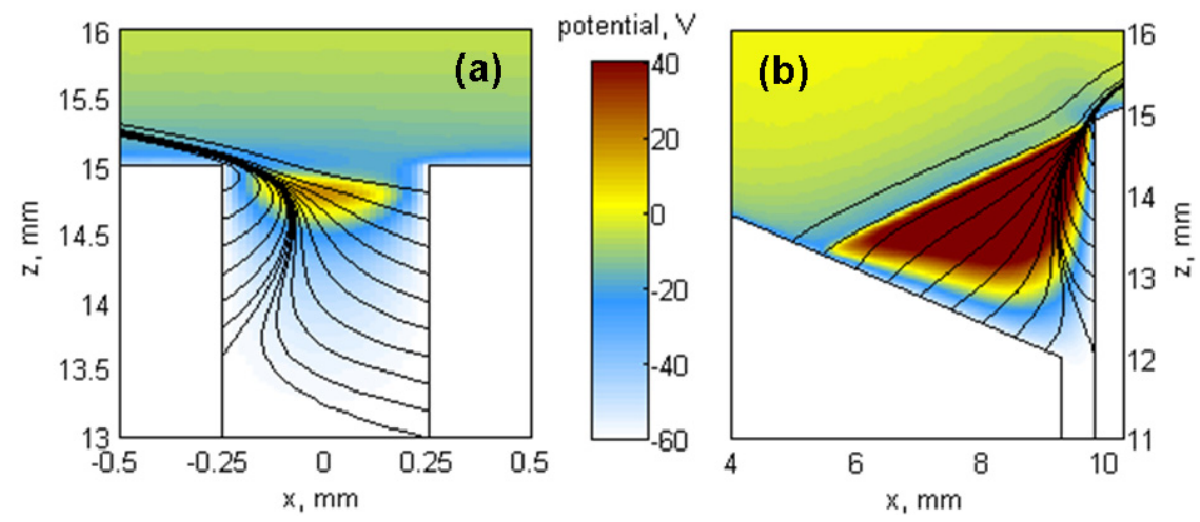

Figure 3. An electrostatic potential map and ion stream lines for the non-shaped (a) and shaped (b) gaps. Please pay attention to different axes scaling in these two cases.

\subsection{Parameter studies}

In the following, the modelling results for different studied cases are validated against the experimental data by comparing the deposition profiles (areal concentrations) of carbon inside the gap. 
In all simulations, $3 \%$ carbon content in the background plasma was assumed as typical for TEXTOR. Figure $4 \mathrm{a}$ and figure $4 \mathrm{~b}$ illustrate how the distances along the gaps are read.

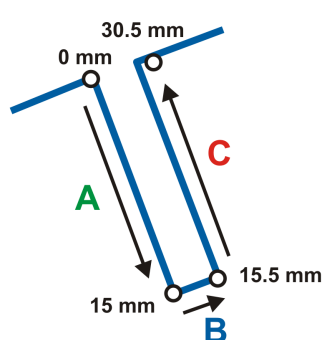

(a)

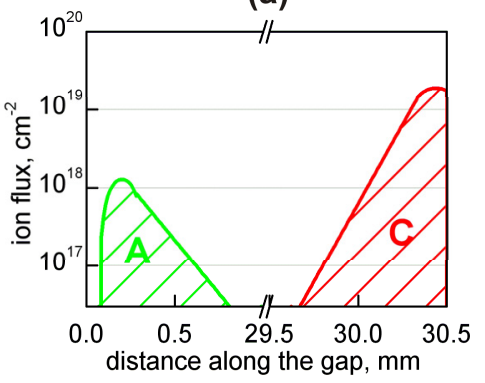

(c)

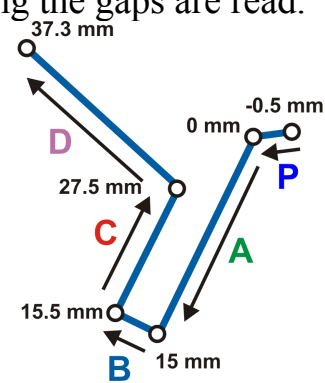

(b)

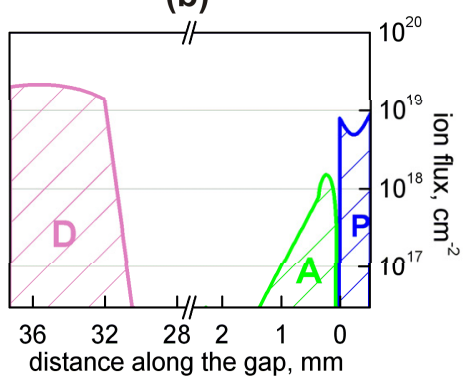

(d)

Figure 4. Way of reading the distances ( $a$ - rectangular gap, $b$ - shaped gap) and distribution of the ion flux ( $\mathrm{c}$ - rectangular gap, $\mathrm{d}$ - shaped gap) along the gaps. Sides denoted by letters $\mathrm{C}$ and $\mathrm{D}$ are called plasma open; sides denoted by letter A are plasma shadowed. Side P is parallel to the magnetic field lines.

3.3.1. Effect of particle reflection. Initially, only background plasma sources were considered, namely carbon ions reflected as neutral atoms from the plasma-wetted areas. Constant reflection coefficient of carbon atoms was used as a free parameter of the model. Modelling results shown in figure 5 illustrate the change of carbon deposition profiles inside the gaps with variation of the reflection coefficient. In the same figure, experimental profiles are shown, for which a constant background was subtracted $\left(\sim 7 \times 10^{16} \mathrm{at} / \mathrm{cm}^{2}\right.$ in case of rectangular gap and $\sim 9 \times 10^{16} \mathrm{at} / \mathrm{cm}^{2}$ in case of shaped gap). For low values of the reflection coefficient, most of particles reside on side walls near the gap entrance; deposition decays very fast down into the gap. With increasing reflection, particles penetrate deeper into the gap. However, the total amount of carbon deposited inside the gap decreases due to loss of particles leaving the gap through the top surface. This is demonstrated in figure 6a where carbon redeposition (ratio of the deposited amount to the amount that entered the gap) in a rectangular gap is plotted against the carbon reflection coefficient. The experimental level of redeposition is estimated on the basis of PIC data for the total ion influx into the gap and assuming $20 \%$ uncertainty for the experimentally measured carbon inventory $\left(3.5 \times 10^{17} a t\right)$. A reflection coefficient of about 0.5 yields very similar values for the carbon amount deposited at the plasma-nearest edge of the plasmashadowed side with corresponding fast decay of deposition over first $2 \mathrm{~mm}$. To recover the slower decay deep in the gap, the presence of highly reflecting species with a reflection coefficient of about 0.9 has to be assumed. Deposition at the bottom of the gap does not change significantly with increasing reflection coefficient and remains well below the experimental level. Accounting for the angular dependence of the reflection coefficient does not change the modelling results substantially. A simple consideration can be the following: For a very narrow and deep gap, geometrically too few particles can reach the bottom even for the case of high reflection. This is illustrated in figure $6 \mathrm{~b}$ where the relative number of test particles reaching the bottom is plotted against the carbon reflection coefficient. For these simulations, a sticking factor 1 at the bottom was assumed which means that all particles reaching the bottom of the gap stick there. In this figure, the range of deposition at the bottom 
of $6-14 \%$ estimated from the experiment is indicated as a hatched area taking into account the estimated level of redeposition (figure 6a).
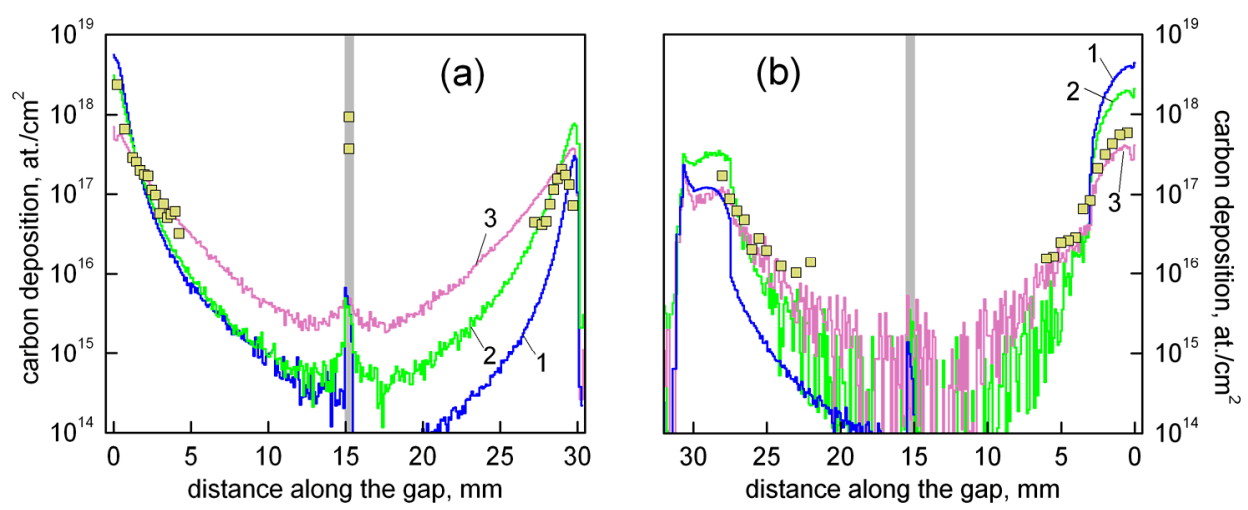

Figure 5. Carbon deposition profiles inside rectangular (a) and shaped (b) gaps for carbon reflection coefficient: 1) $\left.\mathrm{R}_{\mathrm{N}}(\mathrm{C})=0.0 ; 2\right) \mathrm{R}_{\mathrm{N}}(\mathrm{C})=0.5$; and 3) $\mathrm{R}_{\mathrm{N}}(\mathrm{C})=0.9$ in comparison with experimental data (squares). Grey background indicates the gap bottom.
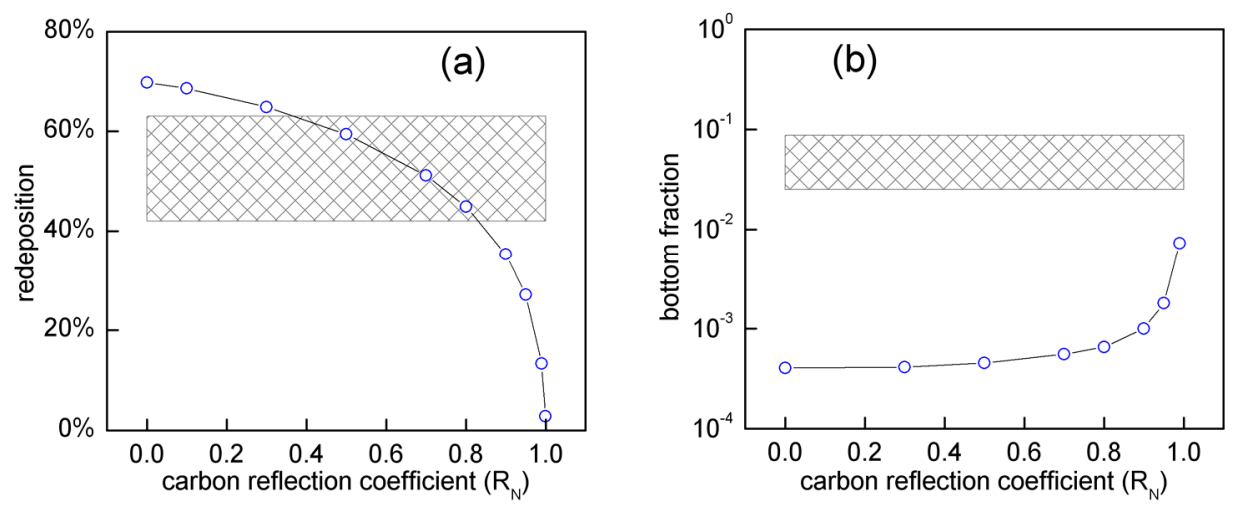

Figure 6. Modelling results for the total amount of carbon redeposited in a rectangular gap (a) and fraction of carbon atoms reaching the bottom of a rectangular gap (b) depending on carbon reflection coefficient $\left(\mathrm{R}_{\mathrm{N}}\right)$. Hatched areas indicate ranges of values estimated from the experiment.

3.3.2 Effect of neutral collisions. If the pressure of $\mathrm{D}_{2}$ gas in the gap is high enough, carbon atoms can suffer from elastic collisions with hydrogen molecules, what might favour the change of the direction of their motion upwards or downwards. Unfortunately, no information on the neutral pressure inside the gap can be obtained experimentally. For a rough estimate of the upper limit of the neutral pressure, one can assume that this pressure is due to deuterium ions and atoms which enter the gap and recycle there forming $\mathrm{D}_{2}$ molecules. The volume concentration of $\mathrm{D}_{2}$ molecules in the gap can be calculated knowing the deuterium influx and its residence time in the gap. This time can be derived under the assumption that deuterium molecules are released with thermal energies and neglecting their residence time in the material. Calculations for the case of the TEXTOR experiment yield a concentration of $\sim 10^{13} \mathrm{~cm}^{-3}$ corresponding to a pressure of $\leq 10^{-2}$ mbar. Simulations have been performed varying the neutral pressure in the range from $10^{-3}$ to 1 mbar (mean free path for collisions from $10^{3}$ to $0.5 \mathrm{~mm}$ ). Results for the case of a carbon reflection coefficient of 0.5 are shown in figure 7 . With an increase of the neutral pressure, more particles leave the gap through the open surface and fewer particles reach the bottom. The total amount of redeposition in the gap decreases. For the estimated pressure values, neutral collisions can be neglected since the mean free path for collisions largely exceeds the gap width. 


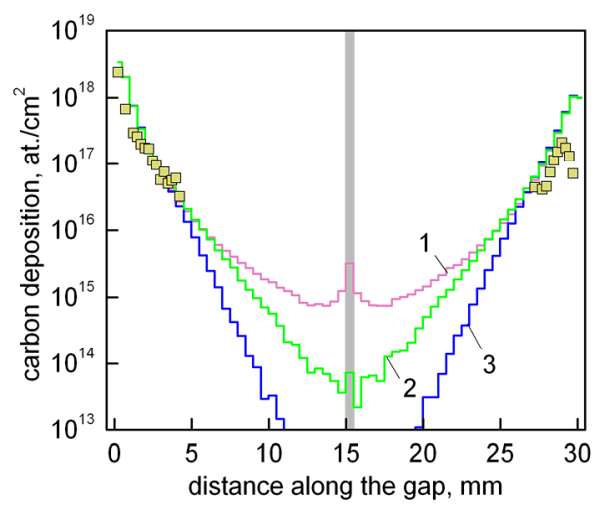

Figure 7. Carbon deposition profiles in a rectangular gap for the neutral pressure in the gap:

1) $p=0.1$ mbar; 2) $p=0.5$ mbar; and 3) $p=1$ mbar in comparison with experimental data (squares).

$\mathrm{R}_{\mathrm{N}}(\mathrm{C})=0.5$. Grey background indicates the gap bottom.

3.3.3. Effect of plasma penetration into the gap. Neutral particles leaving the gap may undergo collisions with plasma ions and electrons. In particular, atoms can become ionized. The effect of ionization by electron impact was investigated using the PIC data of the electron density and temperature. Modelling with the 3D-GAPS code revealed that although non-negligible fraction of particles leaving the gap becomes ionized, it happens mostly well outside the gap and only few of resulting ions have a chance to return into narrow gaps occupying only 5\% of the total top surface of the castellation (only poloidal gaps are considered). Therefore, the carbon deposition profile remains almost unaffected.

3.3.4. Role of particle sources. So far only impurity carbon ions from plasma were considered as source of incoming particles. Neutral carbon atoms resulting from charge-exchange processes may also enter the gap; as well as the break-up products of hydrocarbon radicals eroded from top surfaces of the castellation or escaping from the gap. These neutral particles are not tied to magnetic field lines and, if moving radially, may penetrate deeply into the gap. It is very difficult to access experimentally the value of the neutral carbon flux, so modelling with the 3D-GAPS code has been performed with varying neutral to ion carbon flux ratio. Even for the one to one ratio, deposition at the bottom of the gap hardly reaches $5 \%$ of that near the gap entrance compared with up to $40 \%$ observed in the experiment. Therefore, the described neutral particle sources have no significant effect on the deposition in the gaps in this experiment.

3.3.5. Chemical erosion of redeposited carbon layers. Background plasma particle sources are timeindependent, i.e., particle fluxes are given as input data. In case of surface erosion sources, the erosion yield depends on the surface concentration of the element subject to erosion, which changes with time. The HMM model, as described in section 2.2, was applied to account for surface concentration changes. Modelling runs were performed varying the chemical erosion yield and the effective reflection coefficient of hydrocarbon radicals $\left(\mathrm{C}_{\mathrm{x}} \mathrm{D}_{\mathrm{y}}\right)$ resulting from chemical erosion of carbon by deuterium atoms (everywhere in the gap) and ions (at plasma-wetted areas). To estimate how D atoms reflected from the plasma-wetted area are distributed inside the gap, they were tracked similarly to carbon atoms (with pre-defined reflection coefficient). Additionally, physical sputtering of carbon by plasma ions impinging the plasma-wetted area was taken into account. Modelling results for different values of the chemical erosion yield are presented in figure 8 . Better agreement with experiment is obtained for a high reflection coefficient of hydrocarbons $(\mathrm{R} \geq 0.9)$, which can be attributed to $\mathrm{CH}_{2}$, $\mathrm{CH}_{3}$, and some higher hydrocarbon radicals [20]; and for erosion yields $Y \approx 0.5 \%$ and $Y \approx 2 \%$ in case of rectangular and shaped gaps respectively. For these simulations, reflection coefficients of 0.6 for carbon, 0.7 for deuterium, and 0.9 as effective for hydrocarbons were assumed. The difference in erosion yields may be explained by different plasma fluxes on top surfaces of the cells and hence 
different surface temperatures in conditions of this particular experiment (shaped cells had stronger inclination with respect to magnetic field lines and would be heated stronger). In general, modelling with chemical erosion taken into account is capable of reproducing experimentally observed carbon deposition patterns for side surfaces of the gaps both qualitatively and quantitatively. Exponential decay of the deposit thickness in rectangular gaps with decay length of 1.7-2.0 mm is reproduced. Certain discrepancies still remain for the gap bottom. It is not possible to recover experimentally observed level of deposition at the bottom only by scanning the reflection coefficients of different species and the chemical erosion yield.
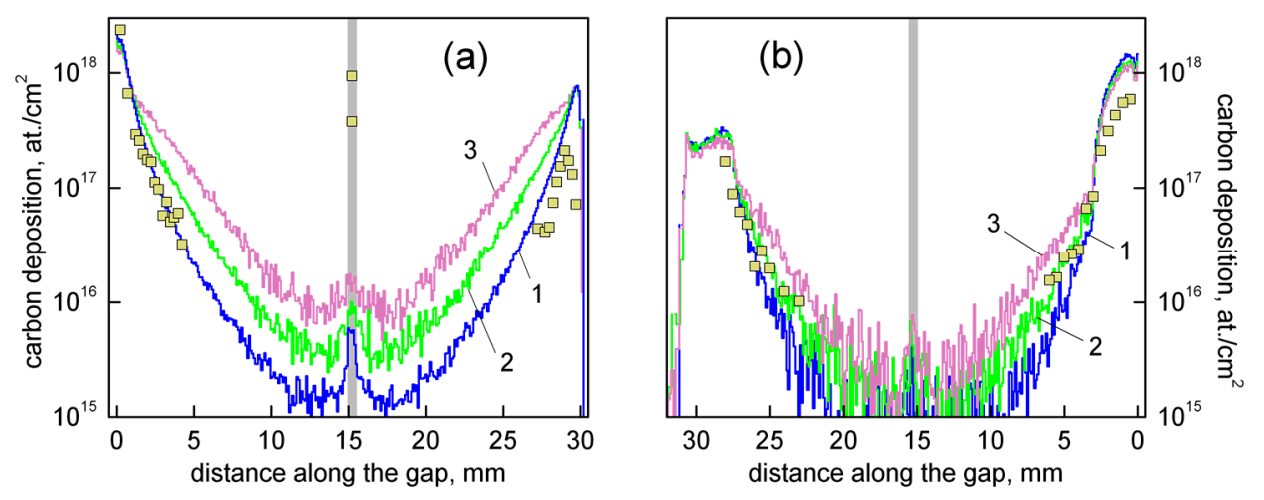

Figure 8. Carbon deposition profiles inside rectangular (a) and shaped (b) gaps for the chemical erosion yield: 1) $\mathrm{Y}=0.5 \%$; 2) $\mathrm{Y}=2 \%$; and 3) $\mathrm{Y}=5 \%$ in comparison with experimental data (squares).

Reflection coefficients are $\mathrm{R}_{\mathrm{N}}\left(\mathrm{C} / \mathrm{D} / \mathrm{C}_{\mathrm{x}} \mathrm{D}_{\mathrm{y}}\right)=0.6 / 0.7 / 0.9$. Grey background indicates the gap bottom.

3.3.6. Angular distribution of reflected particles. For low energies of incident particles, the reflection coefficient may deviate significantly from the cosine distribution assumed so far in the modelling. In particular, if the maximum of the angular distribution is peaked towards the angle of specular reflection, one may expect an increase of particle penetration deep into the gap and deposition at the bottom. However, information on reflection of low energetic particles especially for energies below 1 $\mathrm{eV}$ is barely available. To estimate possible effects of the angular distribution of reflected particles on deposition at the bottom of the gap, modelling with extreme assumptions of specular reflection with $\mathrm{R}_{\mathrm{N}}(\mathrm{C})=0.9$ from side walls of the gap and a sticking factor 1 at the bottom has been performed (without chemical erosion). This allows reproducing the experimentally observed level of deposition at the bottom of the gap (figure 9). Although these extreme assumptions can not be seen as realistic, the importance of the angular distribution of reflected particles can not be neglected. This issue has to be addressed in further studies.

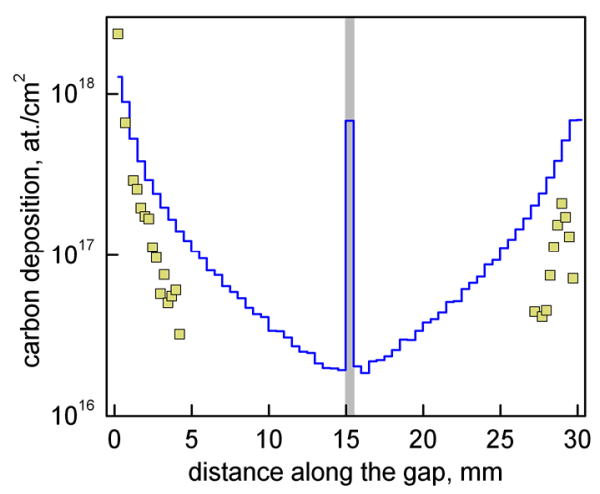

Figure 9. Carbon deposition profile inside the rectangular gap for the extreme case of specular reflection with $\mathrm{R}_{\mathrm{N}}(\mathrm{C})=0.9$ from side walls of the gap and a sticking factor 1 at the bottom in comparison with experimental data (squares). Grey background indicates the gap bottom. 


\section{Summary and outlook}

The 3D Monte-Carlo neutral transport code 3D-GAPS has been developed to model impurity transport and deposition in the gaps of castellated plasma-facing surfaces. The code has been successfully applied to model carbon deposition in poloidal gaps of the castellated test limiter exposed in TEXTOR. The importance of particle reflection and chemical erosion has been revealed. It has been shown that carbon deposition profiles along side surfaces in gaps can be well reproduced qualitatively and quantitatively assuming the presence of species with different reflection probabilities. Carbon impurity ions from the background plasma impinge on the plasma-wetted areas in the gap and are reflected as neutral atoms into the gap. For these carbon atoms low or moderate values of the reflection coefficient are assumed $(\mathrm{R} \leq 0.6)$. Due to deuterium ions and atoms entering the gap, deposited carbon layers can be chemically re-eroded. Assuming a high effective reflection coefficient for produced hydrocarbon radicals $(\mathrm{R} \geq 0.9)$, the experimentally observed decay of deposited amount of carbon deeper into the gap can be reproduced (decay length of 1.7-2.0 mm). Good agreement with experimental data is observed assuming chemical erosion yields of $0.5 \%$ and $2 \%$ for rectangular and shaped gaps, respectively. Elastic neutral collisions and plasma penetration into the gap do not significantly affect the deposition process in the conditions of the modelling. Deposition at the bottom of the gap cannot be reproduced based on similar assumptions for particle sources and reflection properties.

A deviation of the angular distribution of reflected particles from the cosine distribution at low energies is a possible reason for a high bottom deposition level observed in the experiment as it has been demonstrated by modelling with extreme assumption of specular reflection. There were also uncertainties in the experimental conditions such as temperature excursions due to off-normal events (plasma shifts) in the end of exposure. These and other factors such as finite size of the castellation and temperature dependent variation of the chemical erosion yield along the gap surfaces were not taken into account in the modelling and might affect the results. The angular distribution of reflected particles will be addressed in further studies and the particle reflection model will be updated to take into account the energy dependence of the reflection coefficient. Also complete 3D simulations of the castellated structure involving both poloidal and toroidal gaps might help to give more insight into the problem. Such simulations will be possible with a full 3D PIC code being presently developed. Further experiments are planned to clarify the bottom deposition problem. Besides, optimization of the gap geometry in order to reduce the deposition inside gaps is of particular importance. This issue will be addressed by experiments and modelling already in the near future.

Summarizing the data needs for the gap modelling, there is a lack of reliable and systematized data on low energy particle reflection for fusion relevant species including hydrocarbon radicals. Such information usually comes from molecular dynamics simulations and is foreseen to be used in 3DGAPS modelling. A consistent 3D plasma background is also important, which is usually based on modelling with kinetic codes and requires reliable experimental data.

In prospect, the 3D-GAPS code can be used in coupling with ERO in order to improve predictions regarding fuel retention in ITER by taking realistic castellated surfaces into account. There are also other applications planned for the code. Due to its relative flexibility with respect to geometry and particle sources, it is foreseen, in particular, to apply 3D-GAPS to model remote areas such as pump ducts.

\section{Acknowledgements}

This work is supported by the Research Fund of Ghent University (BOF), Belgium. Part of the work was financially supported by Czech Science Foundation, grant 202/07/0044, and is part of the research plan MSM 0021620834 that is financed by the Ministry of Education, Youth and Sports of the Czech Republic.

\section{References}

[1] Loarte A 2007 Nucl. Fusion 47 S203-63

[2] Federici G et al 2001 Nucl. Fusion 41 1967-2137

[3] Roth J et al 2009 J. Nucl. Mater. 390-391 1-9 
[4] Doerner R 2007 J. Nucl. Mater. 363-365 32-40

[5] Kirschner A, Brooks J N, Philipps V, Coad J P and contributors to the EFDA-JET Work programme 2003 Plasma Phys. Control. Fusion 45 309-19

[6] Rubel M J, Coad J P, Wienhold P, Matthews G, Philipps V, Stamp M and Tanabe T 2004 Phys. Scr. T111 112-7

[7] Andrew P et al 1999 Fusion Eng. Des. 47 233-45

[8] Litnovsky A et al 2007 J. Nucl. Mater. 367-370 1481-6

[9] Litnovsky A et al $2009 \mathrm{~J}$. Nucl. Mater. 390-391 556-9

[10] Krieger K et al 2007 J. Nucl. Mater. 363-365 870-6

[11] Rudakov D L et al 2007 Phys. Scr. T128 29-34

[12] Inai K, Ohya K, Tomita Y, Kirschner A, Litnovsky A and Tanabe T 2009 J. Nucl. Mater. 390391 119-22

[13] Federici G, Mayer M, Strohmayer G, Chuyanov V and Day C 2005 J. Nucl. Mater. 337-339 40-4

[14] Kirschner A, Philipps V, Winter J and Kögler U 2000 Nucl. Fusion 40 989-1001

[15] Dejarnac R and Gunn G P 2007 J. Nucl. Mater. 363-365 560-4

[16] Borodin D, Kirschner A, Kreter A, Philipps V, Pospieszczyk A, Ding R, Doerner R, Nishijima D and Yu J 2009 J. Nucl. Mater. 390-391 106-9

[17] Birdsall C K 1985 Plasma Physics via Computer Simulation (New York, McGraw-Hill) p59

[18] ADAS project web page: http://www.adas.ac.uk/

[19] Huber A, Pospieszczyk A, Unterberg B, Brix M, Mertens Ph, Philipps V and Schweer B 2000 Plasma Phys. Control. Fusion 42 569-78

[20] Jacob W 2005 J. Nucl. Mater. 337-339 839-46 